\title{
Peripapillary intrachoroidal cavitation in high myopia: reappraisal
}

Y-H Wei, C-M Yang, M-S Chen, Y-F Shih and $\mathrm{T}-\mathrm{C}$ Ho

\begin{abstract}
Purpose To evaluate optical coherence tomography (OCT) and clinical findings of a peripapillary lesion in high myopia recently named peripapillary detachment in pathologic myopia (PDPM) or intrachoroidal cavitation. Methods Observational case report by chart review, analysis of colour fundus photography, fluorescein angiography, and OCT in 16 eyes of 12 patients with myopic degeneration and the presence of a slightly elevated, patchy peripapillary, yellow-orange lesion on the fundus.
\end{abstract}

Results Patients were mean age 53.1 \pm 10.7 years, with a spherical-equivalent refractive error of $-10.99 \pm 3.33 \mathrm{D}$ and mean axial length of $27.34 \pm 1.44 \mathrm{~mm}$. The mean best-corrected visual acuity was $\log$ MAR $0.3 \pm 0.2$ (between 20/100 and 20/20). Six eyes also showed myopic maculopathy. In 13 eyes, OCT showed an intrachoroidal hyporeflective space located below the normal plane of the retinal pigment epithelium. Six of these $\mathbf{1 3}$ eyes were found to have apparent communication between the intrachoroidal cavity and the vitreous space at the junction of the lesion and the myopic conus. In three eyes, OCT revealed intrachoroidal splitting. The fluorescein angiogram showed early hypofluorescence, without pooling or staining in late phase. Conclusions The yellow-orange elevated patchy lesions adjacent to the peripapillary conus in high myopic eyes may represent either intrachoroidal cavitation or choroidal schisis, which may be different stages of one disease spectrum. Vitreous fluid may be the source of the disruption of choroid and fluid accumulation.

Eye (2009) 23, 141-144; doi:10.1038/sj.eye.6702961; published online 24 August 2007

Keywords: intrachoroidal cavitation; choroidal schisis; high myopia; peripapillary
Introduction

A variety of fundus changes may be seen with increased myopia, including peripapillary conus, posterior staphyloma, lacquer cracks, or chorioretinal atrophy. ${ }^{1}$ Peripapillary detachment in pathologic myopia (PDPM) is a recently recognized peripapillary lesion ${ }^{2}$ characterized by an asymptomatic, localized yellow-orange peripapillary elevation of the retinal pigment epithelium (RPE) and retina. Similar anomalies have been identified by other investigators as intrachoroidal cavitation ${ }^{3}$ or choroidal schisis. ${ }^{4}$ These diverse names suggest the uncertainty of the pathogenesis and pathological changes of this specific clinical change. In this study, we evaluated the clinical and OCT findings of eyes with such a lesion.

Through these findings, we propose the possible pathogenesis of this lesion.

\section{Materials and methods}

We evaluated 16 eyes of 12 myopic patients who were followed-up in the ophthalmology outpatient clinic of National Taiwan University Hospital between 2005 and 2007. The cases were identified, through fundus examination, by their characteristic yellow-orange peripapillary lesion. Optical coherence tomography (Stratus OCT, Carl Zeiss Meditec Inc.) was performed in all eyes. Six-millimetre parallel scans across both the long and short axis of the lesion were carried out; specific attention was paid to the conus-lesion junction. Axial length was measured by A-scan in eight eyes. Visual field was performed in six eyes, and fluorescence angiography was done in two eyes.

\section{Results}

Patients were six men and six women with a mean age of $53.1 \pm 10.7$ years (range: $31-69$ years). All 16 eyes showed myopia degeneration

\section{Department of}

Ophthalmology, National

Taiwan University Hospital, Taipei, Taiwan

Correspondence: C-M Yang, Department of

Ophthalmology,

National Taiwan University

Hospital, No. 7,

Chung Shan South Road,

Taipei, Taiwan

Tel: + 886223123456 ext.

5187;

Fax: + 886223412875

E-mail: chungmay@

ntu.edu.tw

Received: 28 April 2007

Accepted in revised form: 22 July 2007

Published online: 24 August 2007 
with peripapillary atrophy. Four patients had bilateral involvement. The mean spherical-equivalent refraction error was $-10.99 \pm 3.33 \mathrm{D}$ (range: -4.25 to $-16.5 \mathrm{D}$ ).

The mean axial length was $27.34 \pm 1.44 \mathrm{~mm}$ (range: $24.9-29.36 \mathrm{~mm}$ ). The mean best-corrected visual acuity was $\log$ MAR $0.3 \pm 0.2$ (from 20/100 to 20/20). In each patient, a slightly elevated, patchy yellow-orange lesion with indistinct margins was noted bordering on the outer edge of the myopic conus (Figures 1a and 2a). The lesion was located at the inferior border of the myopic conus in five eyes, at the inferiotemporal border in six eyes, at the nasoinferior border in four eyes, and at the superiotemporal border in one eye.

In 13 of 16 eyes (81\%), OCT of the lesion showed intrachoroidal hyporeflective space located below and separated from the normal plane of the RPE (Figure 1d). Six of these 13 eyes were found to have apparent communication between the intrachoroidal cavity and the vitreous space at the point of transition from the yellow-orange lesion to the myopic conus (Figure 1e). In 3 of 16 eyes (19\%), OCT revealed choroidal splitting or choroidal schisis (Figure $2 \mathrm{~b}$ and $\mathrm{c}$ ). The fluorescein angiogram showed early hypofluorescence (Figure 1b) without pooling or staining in late phase (Figure 1c). Six of 16 eyes (37.5\%) also showed myopic maculopathy (Figure 3a). Six eyes (37.5\%) were being treated with drops for glaucomatous visual field defect or thinning of nerve fibre layer.

\section{Discussion}

In a prior case series in which Freund et $a l^{2}$ reported data from 20 eyes with a yellow-orange elevated lesion at the inferior border of the myopic conus, OCT findings were interpreted as indicative of localized RPE detachment and the lesion was designated as PDPM. Toranzo et al ${ }^{3}$ have suggested calling this anomaly peripapillary intrachoroidal cavitation instead of PDPM because OCT

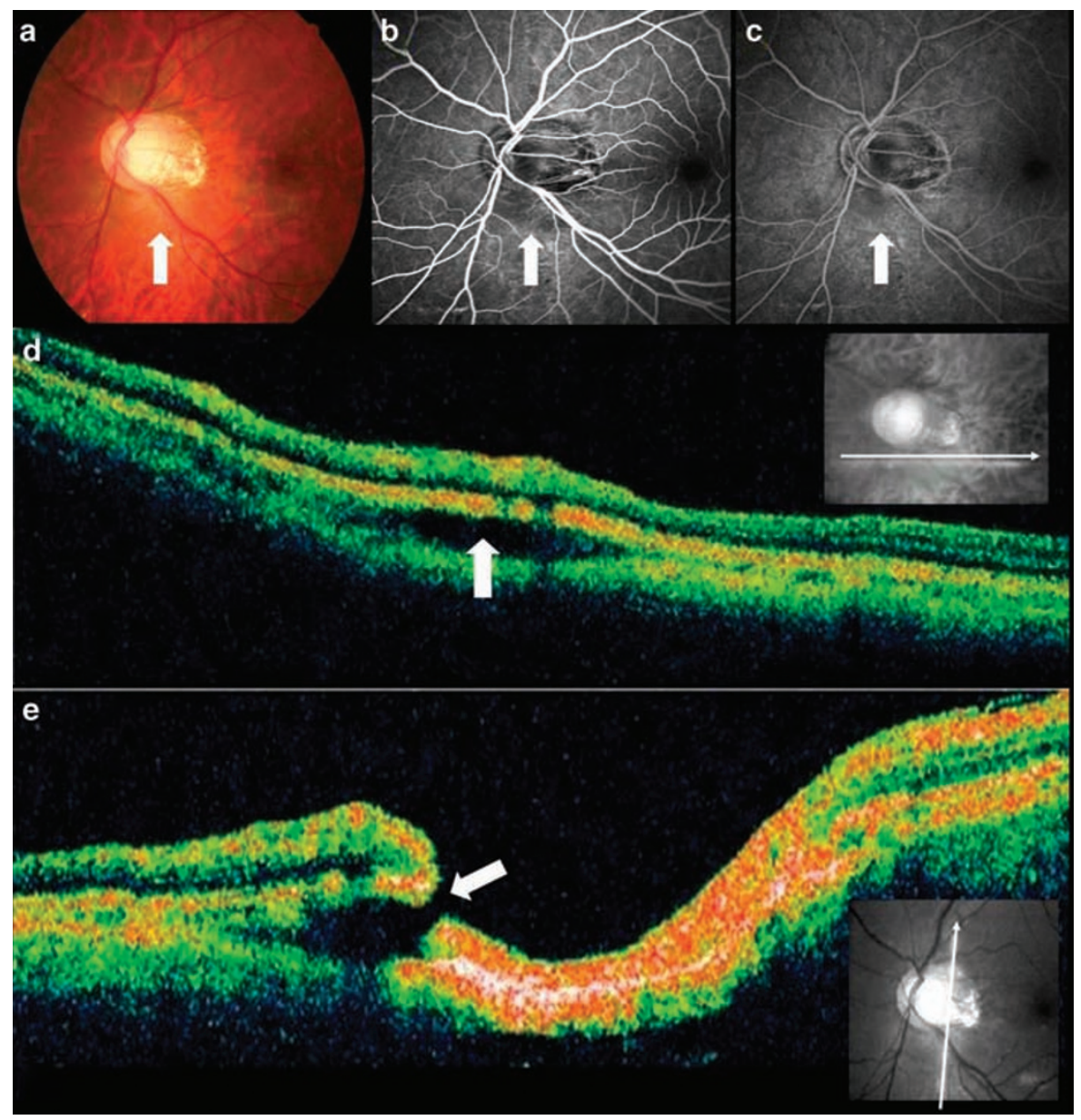

Figure 1 Colour photograph of the left eye of a 37-year-old male (-13.0 D). (a) Yellow-orange lesion seen at inferior border of the myopic conus. (b) Fluorescence angiography showing early hypofluorescence of the lesion and (c) no late-phase pooling or staining of the lesion was noted. (d) OCT showing an intrachoroidal cavitation below normal RPE. (e) Cleft between vitreous and the intrachoroidal space was demonstrated. 


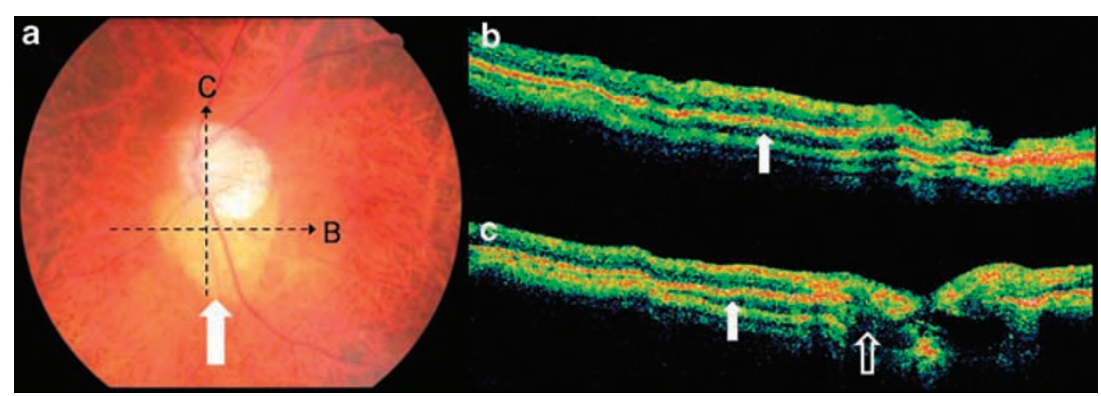

Figure 2 Colour photograph of the left eye of a 69-year-old male (-11.25D). (a) Yellow-orange lesion seen at inferior border of the myopic conus. (b) OCT showing a choroidal schisis-like appearance of the lesion. (c) Choroidal schisis (solid arrow) connected to a pocket-like space (hollow arrow).

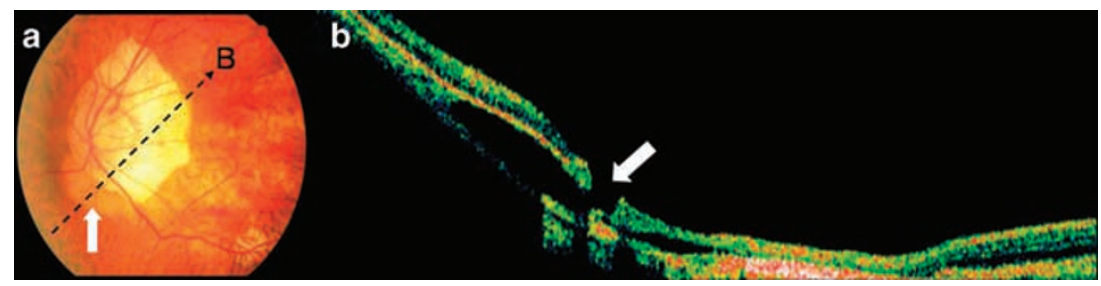

Figure 3 Colour photograph of the left eye of a 58-year-old male (-16.0 D). (a) Yellow-orange lesions seen at nasoinferior border of the myopic conus accompanied by myopic maculopathy. (b) OCT showing intrachoroidal cavitation with a cleft between vitreous and the intrachoroidal space.

showed an intrachoroidal hyporeflective space below the normal RPE plane, but not the well-circumscribed domeshaped elevation typically seen in RPE detachment. In another case series, Hiroki et $a l^{4}$ reported eight eyes with similar findings, but with no separation between RPE and the choroid on the OCT image. They interpreted the lesion as choroidal schisis instead of RPE detachment. The same type of lesion was seen among high myopic eyes, and was identified in 31 (4.9\%) of 362 highly myopic eyes in the study reported by Noriaki et al. ${ }^{5}$

In our study, OCT findings confirmed the presence of peripapillary intrachoroidal cavitation or fluid pocket in high myopic eyes. In addition, intrachoroidal tissue splitting or choroidal schisis without optical empty cavity was found in some cases while schisis adjacent to fluid pocket was found in one case (Figure 2c). Unlike typical features of RPE detachment, fluorescein angiography of the lesion showed early hypofluorescence and late isofluorescence or mild hyperfluorescence. The lack of late intense hyperfluorescence indicates that a change in vascular permeability may not be an important contributor to the fluid accumulation. In six of the eyes on which we report and some cases in previous studies, ${ }^{2-5}$ OCT showed an apparent communication or cleft between the intrachoroidal cavity and the vitreous space. It can be argued as to whether the apparent discontinuity is real or an artefact caused by the perpendicular lining of the thin membrane in relation to the scanning light. We believe there is a true break because repeated scans demonstrated the existence of an intact conus-lesion junctional membrane immediately adjacent to the break.

In our study, the mean spherical-equivalent refraction error was $-10.99 \pm 3.33 \mathrm{D}$ and the mean axial length was $27.34 \pm 1.44 \mathrm{~mm}$. These results are similar to Freund's 12 cases $^{2}$ and Noriaki's 31 cases $^{5}$ in which the mean spherical-equivalent refraction error was -11.0 and $-11.4 \mathrm{D}$ and the mean axial length was 27.4 and $27.7 \mathrm{~mm}$, respectively. The refractive error of all eyes in both Freund's and Noriaki's studies, as well as in most of the eyes in our study, was $>-6.0 \mathrm{D}$. The only exception in our study was a 48 -year-old woman, who had $-4.25 \mathrm{D}$ in the right eye with typical myopic conus and fundus tessellation and in whom the yellow-orange patchy lesion at the inferiotemporal margin of the peripapillary conus was found to be choroidal schisis on the OCT image. There was also a case of $-4.5 \mathrm{D}$ in Hiroko's eight case studies. ${ }^{4}$ We conclude that this peripapillary change is found mostly in highly myopic eyes $(>-6.0 \mathrm{D})$ and also infrequently seen in moderate myopia $>-4.0 \mathrm{D}$.

The cause of the peripapillary intrachoroidal cavitation has been debated. One hypothesis involves the scleral extension around the optic nerve. ${ }^{4}$ Taranzo et $a l^{3,6}$ presumed that the progression of the staphyloma breaks the collagenous limiting tissue of Elschnig between the choroid and the optic nerve, resulting in a retraction of 
the choroid away from the optic nerve margin. We propose that the progression of peripapillary staphyloma may stretch and disrupt the tissue at the edge of the myopic conus and that the adherence of the retina and RPE at the conal margin prevents the break from opening to the subretinal space. Instead, vitreous fluid gradually gains access into the choroidal tissue creating schisis or a fluid pocket. The posterior expansion force, the vitreous traction force, and the vitreous fluid dynamics determine the size and shape of the lesion. We believe that intrachoroidal cavity formation and choroidal schisis may represent different stages of one disease spectrum. Most cases in our study had fluid accumulation in the inferior border of the peripapillary conus. Previous studies also showed only a few cases with lesions located superior to or around the disc. ${ }^{4,5}$ The common location of the lesion suggests a gravitational effect of the vitreous fluid. We were able to identify direct vitreous-fluid pocket communication in only 6 out of 13 cases. This may be due to the fact that there were only small breaks or nonlinear connexions between the vitreous and the fluid pocket.

We saw no direct evidence that the lesion causes a decrease in vision or visual field defect. In Freund's study, during the 6-year follow-up period, the lesion remained stable in most cases $^{2}$ and no apparent negative effect on visual function was noted. However, Noriaki et $a l^{5}$ reported that glaucomatous visual field defects were detected in $71.0 \%$ of patients. In our study, visual field was performed in six eyes, four of which had suspect glaucomatous defects. These four eyes and the other two eyes with thinning of the nerve fibre layer were treated with antiglaucomatous drops even though none of them had intraocular pressure $>21 \mathrm{mmHg}$. The clinical diagnosis of glaucoma is sometimes difficult to make in high myopic eyes because of anomalous optic disc changes and atypical visual field change. Thus, the pathologic significance of the lesion remains to be clarified.
Nishio $e a^{7}$ reported a case of choroidal schisis, which upon initial examination, simulated a choroidal tumour. ${ }^{7}$ Recognition of this lesion is important to distinguish it from other pathologic fundus conditions, such as choroidal tumours or neovascularization, which require further investigation and treatment. OCT is essential in the differential diagnosis.

The results of our study show that when present, the yellow-orange elevated patchy lesions are always adjacent to the peripapillary conus in high myopic eyes and represent either intrachoroidal cavitation or choroidal schisis, which may be the different stages of one disease spectrum. Vitreous fluid may be the source of the disruption of choroid and fluid accumulation. OCT is an essential tool to distinguish this relatively benign lesion from other pathologic conditions.

\section{References}

1 Curtin BJ. The posterior staphyloma of pathologic myopia. Trans Am Ophthalmol Soc 1977; 75: 67-86.

2 Freund KB, Ciardella AP, Yannuzzi LA, Pece A, Goldbaum $\mathrm{M}$, Kokame GT et al. Peripapillary detachment in pathologic myopia. Arch Ophthalmol 2003; 121: 197-204.

3 Toranzo J, Cohen SY, Erginay A, Gaudric A. Peripapillary intrachoroidal cavitation in myopia. Am J Ophthalmol 2005; 140: 731-732.

4 Tateno H, Takahashi K, Fukuchi T, Yamazaki Y, Sho K, Matsumura M. Choroidal schisis around the optic nerve in myopic eyes evaluated by optical coherence tomography. Jpn J Clin Ophthalmol 2005; 59: 327-331.

5 Shimada N, Ohno-Matsui K, Yoshida T, Yasuzumi K, Kojima A, Kobayashi K et al. Characteristic of peripapillary detachment in pathologic myopia. Arch Ophthalmol 2006; 124: 46-52.

6 Anderson DR, Hoyt WF. Ultrastructure of intraorbital portion of human and monkey optic nerve. Arch Ophthalmol 1969; 82: 506-530.

7 Nishio E, Fukushima I, Okada M, Uchida S. A case of choroidal schisis of the macula simulating choroidal tumor. Jpn J Clin Ophthalmol 2006; 60: 1509-1512. 